\title{
Automated Verification of Model Transformations in the Automotive Industry*
}

\author{
Gehan M. K. Selim ${ }^{1}$, Fabian Büttner ${ }^{2}$, James R. Cordy ${ }^{1}$, Juergen Dingel ${ }^{1}$, and \\ Shige Wang ${ }^{3}$ \\ 1 School of Computing, Queen's University, Kingston, Ontario, Canada \\ 2 AtlanMod, École des Mines de Nantes - INRIA, LINA, Nantes, France \\ 3 Electrical and Controls Integration Lab, General Motors Research and \\ Development, Warren, Michigan, USA
}

\begin{abstract}
Many companies have adopted MDD for developing their software systems. Several studies have reported on such industrial experiences by discussing the effects of MDD and the issues that still need to be addressed. However, only a few studies have discussed using automated verification of industrial model transformations. We previously demonstrated how transformations can be used to migrate GM legacy models to AUTOSAR models. In this study, we investigate using automated verification for such industrial transformations. We report on applying an automated verification approach to the GM-to-AUTOSAR transformation that is based on checking the satisfiability of a relational transformation representation, or a transformation model, with respect to well-formedness OCL constraints. An implementation of this approach is available as a prototype for the ATL language. We present the verification results of this transformation and discuss the practicality of using such tools on industrial size problems.
\end{abstract}

Keywords: Model Transformation, Automated Verification, Automotive Industry

\section{Introduction}

Model Driven Development (MDD) has been increasingly used in the last decade for software development and, in many cases, has replaced traditional, codecentric approaches. In MDD, models or software abstractions are the basic building blocks in the software development life cycle and model transformations are the technology used to map between models conforming to different metamodels. Transformations are used for different purposes in MDD, e.g., refactoring, migration, and code generation. Since transformations are essential in MDD, transformation testing and verification is essential to the success of MDD.

\footnotetext{
* This work was partially funded by the Nouvelles Équipes Program of the Pays de la Loire Region (France), and by NSERC (Canada), as part of the NECSIS Automotive Partnership with General Motors, IBM Canada and Malina Software Corp.
} 
Several studies have reported on industrial experiences in adopting MDD [13, 25]. However, only a few of them have specifically discussed using model transformations in industry. Daghsen et al. [14] used transformations to map AUTOSAR timing models to classical scheduling models to perform timing analysis. Giese et al. [15] used triple graph grammars to synchronize SysML system engineering models with AUTOSAR software engineering models. Studies reporting on automated verification of industrial transformations have also been limited.

In this study, we report on using a light-weight, automated verification prototype to reason about the correctness of an ATL [22] transformation developed for the automotive industry [29]. More specifically, we check the correctness of the transformation with respect to OCL well-formedness constraints after translating the ATL transformation into a logical satisfiability problem. The basic approach has been presented in previous work [10] but to our knowledge we are the first reporting on its application to an industrial-sized verification problem.

While the transformation itself is not exceptionally large (in the number of transformation rules), the corresponding metamodels are. Together, they comprise 1586 classes, 897 associations, and 371 multiplicity constraints. Since even types not directly touched by the transformation are relevant for the verification (due to constraints that relate them), we have to deal with large potential instances. To verify our transformation, we have successfully checked models of up to 20000 potential elements with reasonable runtimes (although all counter examples found contained much fewer elements and were found quite quickly). Hence we claim that the verification approach is applicable to realistic verification scenarios.

The rest of this paper is organized as follows: Section 2 gives an overview of the GM-to-AUTOSAR transformation previously presented in [29]; Section 3 introduces the applied verification approach and prototype; Section 4 describes the case study conducted to verify the GM-to-AUTOSAR transformation using the aforementioned prototype; Section 5 summarizes the results of the case study and investigates the performance of the used approach; Section 6 discusses its strengths and limitations; Section 7 summarizes related work in the literature and Section 8 concludes and discusses future work.

\section{Background: Model Transformation in the Automotive Industry}

We now review the GM-to-AUTOSAR transformation presented in [29] which was used to migrate GM legacy models to the AUTOSAR standard.

\subsection{Overview of the Model Transformation Problem}

As one of the leading automotive companies, General Motors has been adopting MDD for the develoment of automotive software. GM engineers have been using a domain-specific metamodel for the development of vehicle control software (VCS). We refer to their domain-specific metamodel as the GM metamodel. 
AUTOSAR (the AUTomotive Open System ARchitecture) [2] has been developed and adopted by many organizations as an automotive industry standard that is meant to facilitate the development and integration of software components from different vendors. AUTOSAR specifies requirements for software that is meant to conform to the standard. Further, AUTOSAR has its own metamodel with a well-defined architecture and interfaces.

Since the majority of organizations in the automotive industry are migrating to AUTOSAR, transforming models conforming to the GM metamodel to their equivalent AUTOSAR models is an important goal. Thus, we have previously developed and reported on a transformation that maps between subsets of the GM metamodel and the AUTOSAR metamodel as its source and target metamodels. In that work, we focused on subsets of the two metamodels that represent the deployment and interaction of software components.

\subsection{The GM Metamodel}

Fig. 1 illustrates the subset of the GM metamodel that we manipulated in our transformation in $[29]^{1}$. The PhysicalNode models a physical node on which soft-

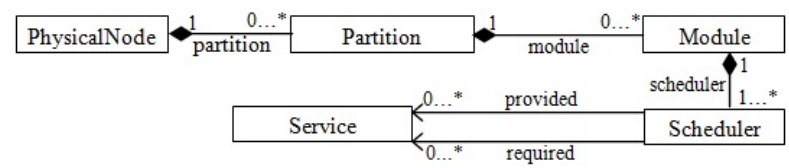

Fig. 1. Subset of the GM metamodel directly used by our transformation in [29].

ware is deployed. A PhysicalNode may contain multiple Partitions (i.e., processing units or memory partitions) on which software is deployed. Multiple Modules can be deployed on a single Partition. A Module is an atomic, deployable, and reusable element in a product line and can contain multiple Schedulers. A Scheduler is the basic unit for software scheduling. It contains behavior-encapsulating entities, and is responsible for managing services provided or required by the behavior-encapsulating entities. Each Scheduler may provide and/or require Services, which model the services provided or required by the Scheduler.

\subsection{The AUTOSAR Metamodel}

The AUTOSAR metamodel is defined as a set of templates. Each template specifies an AUTOSAR artifact such as software components. Among the defined templates, the System template [1] models the configuration of a system or an Electronic Component Unit (ECU). An ECU is a physical unit on which software is deployed. When used for modeling the configuration of an ECU, the System template is referred to as the ECU Extract. Fig. 2 shows the subset of the ECU Extract manipulated by our transformation. The ECU extract is modeled using the System type that aggregates SoftwareComposition and SystemMapping

\footnotetext{
${ }^{1}$ In this study, we follow the same obfuscated naming conventions that we used for the GM metamodel in [29] for reasons of confidentiality.
} 


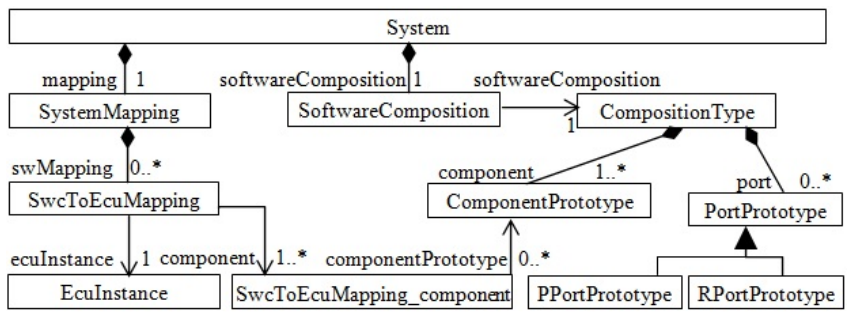

Fig. 2. Subset of the AUTOSAR System Template directly used by our transformation.

elements. The SoftwareComposition type points to the Composition Type type which eliminates any nested software components in a SoftwareComposition. The SoftwareComposition type models the architecture of the software components deployed on an ECU, the ports of these software components and the ports' connectors. Each Software component is modeled using the ComponentPrototype type, which defines the structure and attributes of a software component; each port is modeled using the PortPrototype type (i.e., a PPortPrototypeor a $R$ PortPrototype) for providing or requiring data and services.

The SystemMapping type binds the software components to ECUs and the data elements to signals and frames (not shown). The SystemMapping type aggregates the SwcToEcuMapping type, which assigns SwcToEcuMapping_components to an EcuInstance. SwcToEcuMapping_components in turn, refer to ComponentPrototype elements. According to AUTOSAR, only one SwcToEcuMapping should be created for each processing unit or memory partition in an ECU.

\section{Verification Methodology}

We apply the automated verification approach presented in [10] to the GM-toAUTOSAR transformation. In short, we translate the ATL transformation $T$, its source metamodel $M M_{s r c}$, and its target metamodel $M M_{t a r}$ into a combined model, or a transformation model, consisting of $M M_{s r c}$ and $M M_{t a r}$ and additional model elements that represent the transformation rules. Additionally, a set Sem of OCL constraints is generated for the combined model that characterizes the execution semantics of the ATL rules. For declarative ATL rules without recursion, the constraints describe the ATL semantics one-to-one, i.e., each valid instance of the transformation model corresponds to an execution of the transformation and vice versa.

Using this representation we can check partial correctness of the transformation with respect to properties specified as OCL constraints over the source and/or the target model, by checking if there exists a counterexample within a specific scope (i.e., maximum number of objects per class). More specifically, for a set of transformation preconditions (or assumptions) Pre $_{1}, \ldots$, Pre $_{n}$ and a set of postconditions (or assertions) Post $_{1}, \ldots$, Post $_{m}$, we want to show that for 
each instance $M$ of the transformation model,

$$
\begin{aligned}
& \left(S_{e} m_{1} \text { and } S_{e m} \text { and } \ldots \text { and } S e m_{k}\right. \text { and } \\
& \text { Pre }_{1} \text { and } \text { Pre }_{2} \text { and ... and } \text { Pre }_{n} \text { ) implies }
\end{aligned}
$$

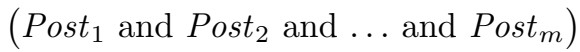

holds. This can be expressed equivalently as follows: For each postcondition Post $_{i}(1 \leq i \leq m)$, the following formula must be unsatisfiable (i.e., there is no model $M$ under which the formula is true):

$$
S e m_{1} \text { and } \ldots \text { and } \text { Sem }_{k} \text { and Pre } e_{1} \text { and } \ldots \text { and Pre }{ }_{n} \text { and not }\left(\text { Post }_{i}\right)
$$

Fig. 3 illustrates this using a simple example. In the upper part we have an ATL transformation (c) over the shown source and target metamodels (a) and (b). The transformation copies the A-B structure to the C-D structure, but creates an additional D object when copying an 'empty' A object. The middle part shows the transformation model of this transformation. In the class diagram (d), each of the three rules is translated into a trace class and connected to the source and target classes according to the from and to patterns of the rule. The OCL constraints (e) capture the execution semantics of the transformation such as the matching of rule R1, the binding of primitive and object-typed properties, and the controlled creation of target objects. Some pre-/post- conditions are shown in (f) and (g), respectively.

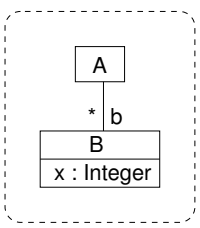

(a) Source MM

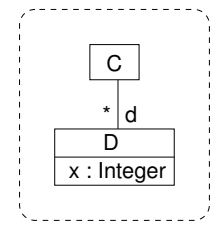

(b) Target MM

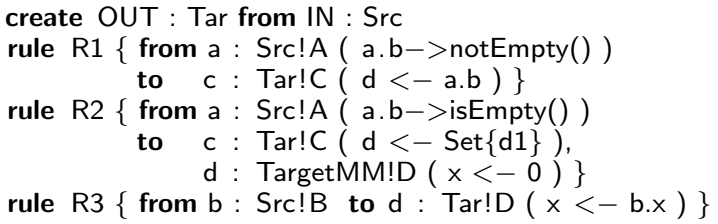

(c) ATL transformation

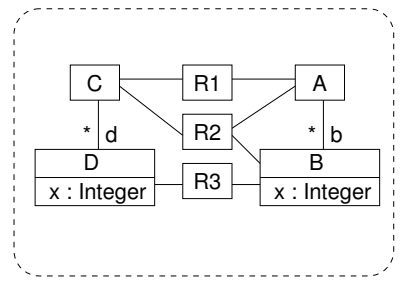

(d) Transformation model

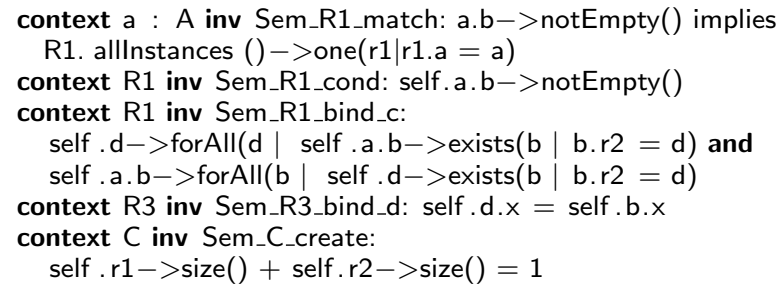

(e) OCL constraints for ATL semantics (excerpt) context $A$ inv Pre1: self .b. $x->\operatorname{sum}()>=0$ context $A$ inv Pre2: self $. b->\operatorname{size}()>=1$

(f) Preconditions context $C$ inv Post1: self . $d->\operatorname{size}()>=1$ context $C$ inv Post2: self . $b . x->\operatorname{sum}()>=0$

(g) Postconditions

Fig. 3. Transformation model example 
To verify that, for example, postcondition Post $_{i}$ is implied by the transformation (given the preconditions), we have to check that Eq. (2) is unsatisfiable. This can be tested using metamodel satisfiability checkers, or model finders, such as the USE Validator [23] which is publicly available [35]. The USE Validator translates the UML model and the OCL constraints into a relational logic formula and employs the SAT-based solver Kodkod [33] to check the unsatisfiability of Eq. (2) for each of the post-conditions Post $t_{i}$ within a given scope. Thus, we have four different representations of the problem space, (i) ATL + OCL, (ii) OCL, (iii) relational logic, and (iv) propositional logic (for the SAT solver).

We have implemented the whole chain as an verification prototype (Fig. 4). We have implemented the ATL-to-OCL transformation [10] as a higher-order ATL transformation [32], i.e., a transformation from Ecore and ATL metamodels to Ecore metamodels (where the Ecore model can contain OCL constraints as annotations). Our implementation automatically generates the Sem constraints from the ATL transformation as well as Pre and Post constraints from the structural constraints in the source and target metamodels (further constraints to be verified can be added manually). Since the USE validator has a proprietary metamodel syntax, we have created a converter from Ecore to generate a USE specification. We also generate a default search space configuration, which is a file specifying the scopes and ranges for the attribute values. In the search configuration, we can disable or negate individual invariants or constraints.

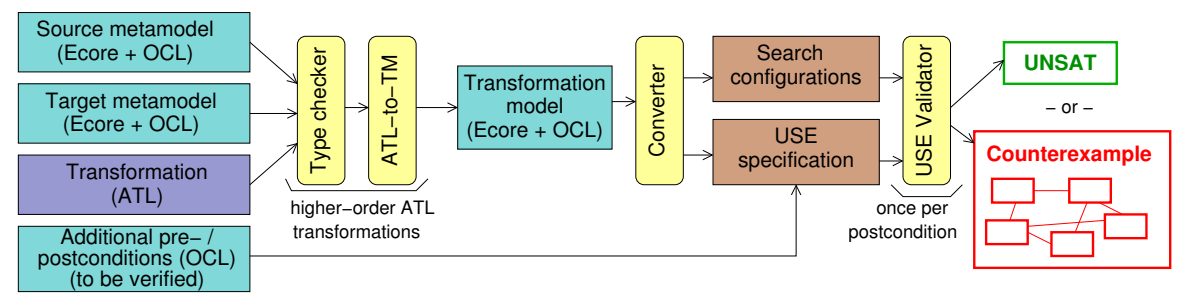

Fig. 4. The tool chain used to perform the transformation verification.

Steps to verify a postcondition using the prototype: To check Eq. (2) for a postcondition, we have to negate the respective postcondition and disable all other postconditions in the generated search configuration (Fig. 4) and then run USE. If USE reports 'unsat', this implies that there is no input model in the search space for which the transformation can produce an output model that violates the postcondition. If there exists a counterexample, USE provides the object diagram of the counterexample which can be analyzed using many browsing features of the tool. Although the implementation is a prototype, it is not specific to the GM-to-AUTOSAR transformation.

\section{Case Study: Evaluating Transformations in the Automotive Industry Using Automated Verification}

We use the prototype described in Section 3 to verify our GM-to-AUTOSAR transformation. However, the verification prototype can only verify ATL transformations composed of declarative matched rules and non-recursive lazy rules. 
Thus we have changed the implementation described in [29] to be completely declarative and compatible with the format required by the prototype. The final reimplementation is intended to achieve the same mapping as the original implementation described in [29].

In this section, we describe the constructs used to re-implement our transformation and the different kinds of constraints formulated for verification.

\subsection{Reimplementation of the GM-to-AUTOSAR Model Transformation}

In the first implementation of the GM-to-AUTOSAR transformation, we used two ATL matched rules, 9 functional helpers and 6 attribute helpers to implement the required mapping between the two metamodels. After reimplementing the transformation to be completely declarative, the new transformation was composed of three matched rules and two lazy rules. Although we had to reimplement the transformation to use the verification prototype, we point out that the new declarative implementation is simpler and more readable. The rules implemented are listed in Table 1 together with the types of the rules, the input element matched by the rule, and the output elements generated by the rule.

\begin{tabular}{|l|l|l|l|}
\hline Rule Type & Rule Name & Input Types & Output Types \\
\hline Matched Rule & createComponent & Module & $\begin{array}{l}\text { SwComp ToEcuMapping_component, } \\
\text { ComponentPrototype }\end{array}$ \\
\hline Matched Rule & initSysTemp & PhysicalNode & $\begin{array}{l}\text { System, SystemMapping, SoftwareCom- } \\
\text { position, CompositionType, EcuInstance }\end{array}$ \\
\hline Matched Rule & initSingleSwc2EcuMapping & Partition & SwcToEcuMapping \\
\hline Lazy Rule & createPPort & Scheduler & PPortPrototype \\
\hline Lazy Rule & createRPort & Scheduler & RPortPrototype \\
\hline
\end{tabular}

Table 1. The types of ATL constructs used to reimplement the transformation, their designated names, and their input and output element types.

As described in [29], the relationships between the outputs of the matched rules are built using the ATL predefined function resolveTemp. The resolveTemp function allows a rule to reference the elements that are yet to be generated by another rule at runtime. For example, the resolveTemp function was used to connect the SwcToEcuMapping elements created by the initSingleSwc2EcuMapping matched rule to the SystemMapping element created by the initSysTemp matched rule. Further, the matched rule initSysTemp calls the two lazy rules and assigns the union of the lazy rules' outputs to the ports of the Composition Type produced by the initSysTemp rule.

\subsection{Formulation of OCL Pre- and Postconditions}

In general, the OCL postconditions in our approach can be either defined on elements of the target metamodel only (then we call them target invariants), or they can relate the elements of the source and target metamodels (then we call them transformation contracts). Usually, a transformation contract specifies an 
implication 'when an input has a property then it's corresponding output has a property'. The OCL preconditions are propositions about the input that we assume to always hold.

In our case study, the preconditions were given by the multiplicity and composition constraints automatically extracted from the GM metamodel as OCL constraints. The formulated OCL postconditions are summarized in Table 2. We divide the formulated postconditions into four categories: Multiplicity Invariants, Uniqueness Contracts, Security Invariants, and Pattern Contracts. For each constraint in Table 2, we add to the beginning of its formulation an abbreviation (e.g., $(M 1),(U 2)$ ) that will be used in the rest of the paper to refer to the constraint. The Multiplicity Invariants were automatically generated by the prototype. All the other postconditions were manually formulated.

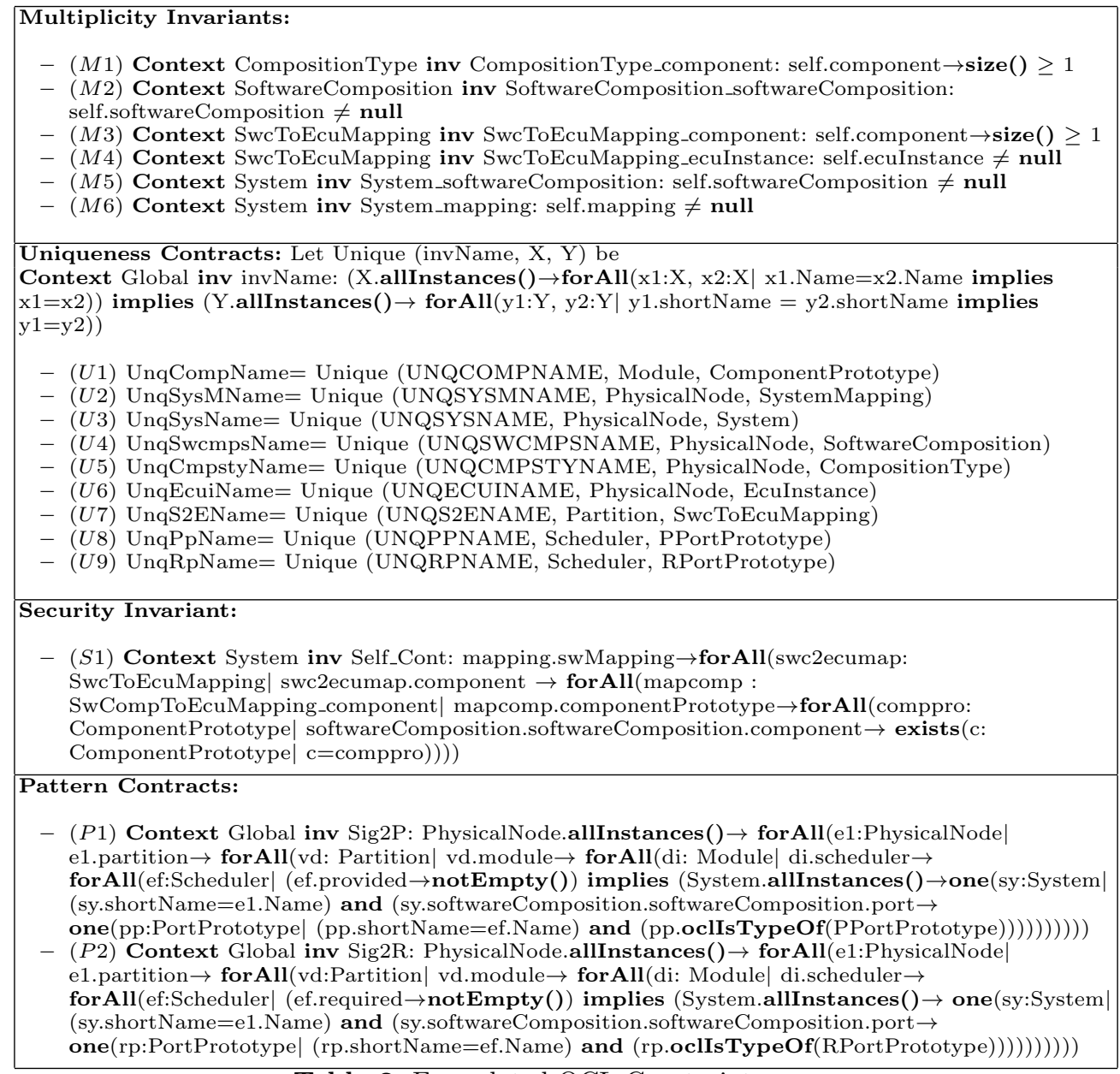

Table 2. Formulated OCL Constraints 
Multiplicity Invariants ensure that the transformation does not produce an output that violates the multiplicities in the AUTOSAR metamodel (Fig. 2). As described in Section 3, the prototype generates a USE specification with a multiplicity invariant for each multiplicity in the AUTOSAR metamodel. Ideally, we would check the satisfiability of all the multiplicity invariants generated for the AUTOSAR metamodel. Since our transformation manipulates a subset of the metamodels, we only check multiplicity invariants for output elements affected by our transformation. We have identified six of the generated multiplicity invariants that are affected by our transformation. ( $M 1)$ ensures that each $\mathrm{Com}$ position Type is associated to more than one ComponentPrototype through the component association. (M2) ensures that each SoftwareComposition is associated with one Composition Type through the softwareComposition association. The rest of the multiplicity invariants can be interpreted in a similar way.

Uniqueness Contracts require the output element (of type Y) generated by a rule to be uniquely named (by the shortName attribute) within its respective scope if the corresponding input element (of type X) matched by the rule is uniquely named (by the Name attribute) within its scope too. For example, in Section 4.1, we discussed that the matched rule createComponent maps Modules to ComponentPrototypes. Thus, U1 mandates that the ComponentPrototypes generated by the transformation are uniquely named, if the corresponding Modules are uniquely named too. The rest of the uniqueness contracts are similar and ensure uniqueness of the output elements of each rule described in Section 4.1 if their corresponding input elements are unique too.

The only security invariant defined, $S 1$, mandates that within any System element, all its composite SwcToEcuMappings must refer to ComponentPrototypes that are contained within the CompositionType lying under the same System element (refer to Fig. 1). Thus, this invariant assures that any ECU configuration (modeled by a System element) is self contained and does not refer to any ComponentPrototype that is not allocated in that ECU configuration.

Pattern contracts require that if a certain pattern of elements is found in the input model, then a corresponding pattern of elements must be found in the output model. Pattern contracts also mandate that corresponding elements in the input and output patterns must have the same name. P1 mandates that if a PhysicalNode is connected to a Service through the provided association (in the input model), then the corresponding System element will eventually be connected to a PPortPrototype. P1 also ensures that the names of the PhysicalNode and the System are equivalent and that the names of the Scheduler (containing the Service) and the PPortPrototype are equivalent. The contract P2 is similar to $P 1$ but manipulates required Services and RPortPrototypes instead.

Since invariants are constraints on target metamodel elements, the Multiplicity and Security invariants are specified within the context of their respective AUTOSAR elements. Since contracts are constraints on the relationships between the source and target metamodel elements, they do not relate to an AUTOSAR element per se. Thus, we add a class to the USE specification file, Global, which is used as the context of the Uniqueness and Pattern contracts. 


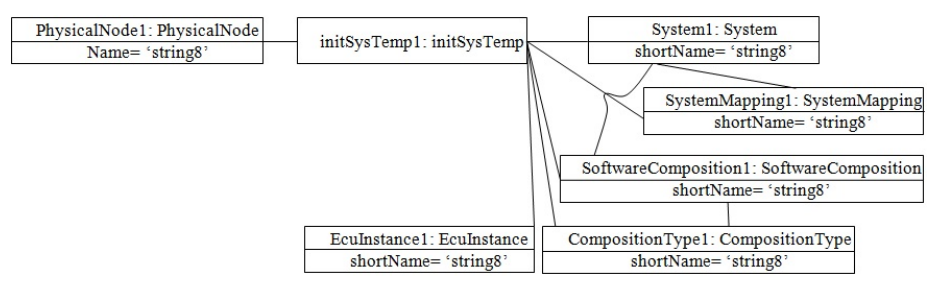

Fig. 5. Counterexample generated for the mult. inv. CompositionType_component.

\section{Results}

In this section, we discuss the results of verifying the OCL contraints defined in Section 4.2 using the verification prototype. We show how the verification prototype was able to uncover bugs in the GM-to-AUTOSAR transformation that were fixed and re-verified. We also describe the results of a study to determine the performance of the used verification approach.

\subsection{Verifying the Formulated OCL Constraints}

Using the verification prototype, we generated a USE specification and a search configuration as shown in Fig. 4. After adding the constraints (Table 2) to the USE specification, we ran the USE tool once for each constraint.

Out of the 18 constraints defined in Table 2, two multiplicity invariants were found to be violated by the transformation: CompositionType_component and SwcToEcuMapping_component. In other words, our transformation can generate a Composition Type with no ComponentPrototypes and/or a SwcToEcuMapping with no ComponentPrototypes. Both of these possible outputs violate the multiplicities defined in the AUTOSAR metamodel (Fig. 2). The counterexamples were found by USE even within a scope of just one object per concrete class.

Due to the page limit, we only show an excerpt of the counterexample generated for the invariant CompositionType_component in Fig. 5. The counterexample shows that the rule initSysTemp maps a PhysicalNode to five elements, one of which is CompositionType. Since the rule does not have any restrictions on the generated Composition Type, it was created without associating it to any ComponentPrototype through the component association. The counterexample for the SwcToEcuMapping_component invariant was similar showing that the initSingleSwc2EcuMapping rule creates a SwcToEcuMapping element without mandating that it is associated to any SwCompToEcuMapping_component element through the component association.

After examining the two counterexamples generated by USE for the two violated multiplicity invariants, we identified two bugs in two rules shown in Table 3: initSysTemp and initSingleSwc2EcuMapping. The bold, underlined text are the updates to the rules that fix the two bugs. initSysTemp initially mapped a PhysicalNode to many elements, including a CompositionType that must contain at least one ComponentPrototype. If the PhysicalNode did not have any Module in any of its Partitions, then the created Composition Type will not contain any ComponentPrototypes. Thus we added a matching constraint 


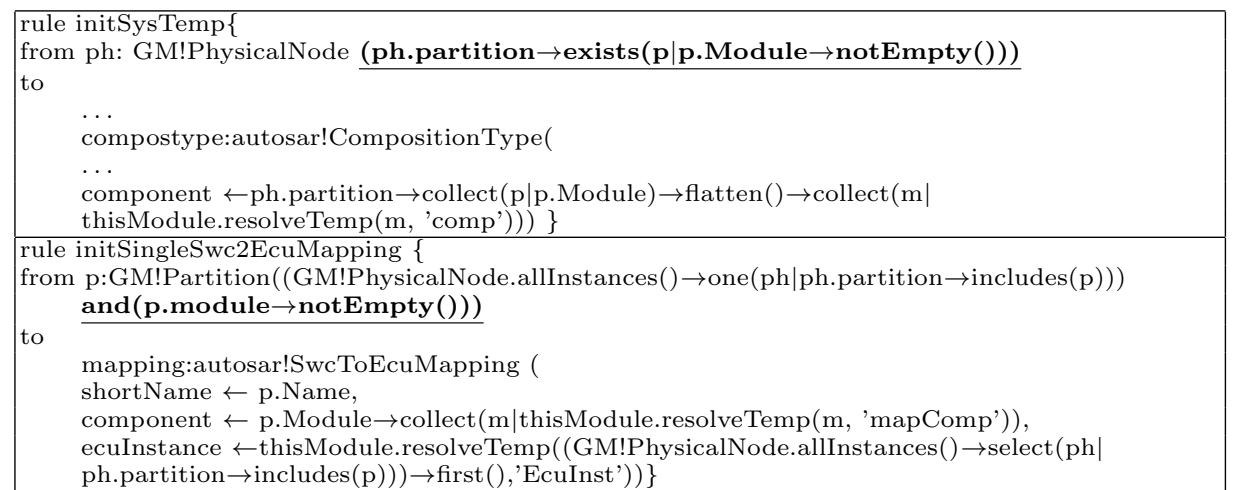

Table 3. The two rules that required updates to address the two violations of multiplicity invariants.

to the PhysicalNode matched by the rule to ensure that any of its Partitions must contain at least one Module. Similarly, initSingleSwc2EcuMapping initially mapped a Partition to a SwcToEcuMapping that must contain at least one SwCompToEcuMapping_component. If the Partition did not have any Module, then the created SwcToEcuMapping will not contain any SwCompToEcuMapping_component. Thus we added a matching constraint to the Partition matched by the rule to ensure that it must contain at least one Module.

The 18 constraints were reverified on the updated transformation, and were all found to be satisfied.

\subsection{Performance of the Verification Approach}

To explore the performance of our approach, we used the verification prototype to verify the 18 constraints (Table 2) for different scopes. We ran the verification with scopes between one and 12 . We only show the results for scopes $6,8,10$, and 12 due to the page limit. The scope determines the maximum number of objects per concrete class in the search space. In our tests, we used the same scope for all classes, although it could be set individually. Since our transformation model has 1586 classes, a scope of $n$ generates a model with $1586 n$ potential elements (and their corresponding links and attribute values). All experiments where run on a standard laptop at $2.50 \mathrm{GHz}$ and $16 \mathrm{~GB}$ of memory, using Java 7, Kodkod 2.0, and Glucose 2.1.

For each combination of constraint and scope, the prototype generates two time values: the time the prototype takes to translate the relational logic formula into a propositional formula (i.e., translation time) and the time the SAT solver takes to solve the formula (i.e., constraint solving time).

We show these two time values (in seconds) in Table 4. Each column represents the time intervals for each of the 18 constraints, where the Constraint Abbreviation is the abbreviation given to each constraint in Table 2 (e.g., (M1) and (U5)). Each row represents the time intervals for a different scope. Thus, 
each cell within the table shows the translation time and the constraint solving time of a certain constraint at a specific scope.

\begin{tabular}{|c|c|c|c|c|c|c|c|c|c|c|}
\hline & \multicolumn{9}{|c|}{ Constraint Abbreviation (from Table 2) } \\
\hline & & U1 & $\mathrm{U} 2$ & U3 & U4 & U5 & U6 & U7 & U8 & U9 \\
\hline \multirow{4}{*}{ 莒 } & 6 & $76 \backslash 25$ & $76 \backslash 19$ & $76 \backslash 22$ & $76 \backslash 7$ & $77 \backslash 19$ & $76 \backslash 24$ & $76 \backslash 7$ & $76 \backslash 7$ & $74 \backslash 5$ \\
\hline & 8 & $169 \backslash 74$ & $165 \backslash 79$ & $168 \backslash 106$ & $165 \backslash 37$ & $168 \backslash 85$ & $171 \backslash 68$ & $167 \backslash 38$ & $166 \backslash 57$ & $169 \backslash 45$ \\
\hline & 10 & $279 \backslash 165$ & $280 \backslash 188$ & $279 \backslash 210$ & $281 \backslash 114$ & $277 \backslash 211$ & $280 \backslash 207$ & $281 \backslash 147$ & $282 \backslash 170$ & $279 \backslash 206$ \\
\hline & 12 & $455 \backslash 976$ & $434 \backslash 643$ & $431 \backslash 623$ & $428 \backslash 322$ & $426 \backslash 827$ & $428 \backslash 616$ & $425 \backslash 584$ & $427 \backslash 604$ & $430 \backslash 501$ \\
\hline
\end{tabular}

\begin{tabular}{|c|c|c|c|c|c|c|c|c|c|c|}
\hline & \multicolumn{9}{|c|}{ Constraint Abbreviation (from Table 2) } \\
\hline & & M1 & M2 & M3 & M4 & M5 & M6 & S1 & P1 & $\mathbf{P 2}$ \\
\hline \multirow{4}{*}{ : } & 6 & $74 \backslash 2$ & $73 \backslash 0.4$ & $74 \backslash 1$ & $74 \backslash 1$ & $75 \backslash 0.5$ & $74 \backslash 0.5$ & $74 \backslash 40$ & $242 \backslash 14$ & $244 \backslash 7$ \\
\hline & 8 & $162 \backslash 2$ & $162 \backslash 1$ & $164 \backslash 2$ & $163 \backslash 2$ & $164 \backslash 1$ & $166 \backslash 1$ & $168 \backslash 429$ & $1453 \backslash 37$ & $1422 \backslash 65$ \\
\hline & 10 & $280 \backslash 12$ & $281 \backslash 1$ & $277 \backslash 6$ & $281 \backslash 3$ & $275 \backslash 1$ & $274 \backslash 1$ & $277 \backslash 3619$ & $6225 \backslash 80$ & $6178 \backslash 249$ \\
\hline & 12 & $426 \backslash 18$ & $425 \backslash 1$ & $421 \backslash 25$ & $424 \backslash 4$ & $422 \backslash 1$ & $425 \backslash 1$ & * & $21312 \backslash 710$ & $21092 \backslash 814$ \\
\hline
\end{tabular}

Table 4. Translation $\backslash$ Constraint Solving times (seconds) for the 18 constraints on different scopes. For a scope of 12, the verification of S1 did not terminate in a week.

Two observations can be made from Table 4. First, despite the exponential complexity of checking boolean satisfiability, we could verify the postconditions for scopes up to 12 in most of the cases; only the analysis of S1 did not finish for scope 12; the constraint solving time of S1 in scope 10 was the longest (just over an hour). Although we have no proof that no bugs will appear for bigger scopes, we are confident that a scope of 12 was sufficient to uncover any bugs in our transformation with respect to the defined constraints. In fact, the two bugs that were uncovered and fixed were found at a scope of one.

Second, the translation times are larger than expected and grow mostly polynomially. This can be attributed to the approach used by Kodkod to unfold a first-order relational formula into a set of clauses in conjunctive normal form (CNF), given an upper bound for the relation extents [33]. While transforming a formula into CNF grows exponentially with the length of the formula, it only grows polynomially with the scope in our case (as the formula's length does not change significantly). For example, each pair of nested quantifiers will generate a number of clauses that grows quadratically with the scope. The relational logic constraints generated implicitly by USE for all associations expand similarly. This justifies why the two pattern contracts (i.e., P1 and P2) show the highest translation times; they have the most quantifiers of the 18 constraints.

Using an incremental SAT solver would improve the performance of the prototype. Since most of the generated Boolean formula is the same for all the 18 constraints (i.e., the encoding of classes, associations, multiplicities, and preconditions), we expect that the translation (i.e., the first number in each cell of Table 4) can be done once for the entire verification process; except for $P 1$ and $P 2$ which differ in their high number of nested quantifiers.

\section{Discussion}

\subsection{Strengths of the Verification Approach}

We claim that the verification approach is practical to use for two reasons. First, the used approach provides a fully automated translation from ATL transformations and their constrained metamodels to OCL and relational logic. The 
approach further provides a fully automated verification of the generated translation. Even when applied to a realistic case study, the approach scaled to a scope that was large enough to strongly suggest that the analysis did not overlook a bug in the transformation due to the boundedness of the underlying satisfiability solving approach. If we wanted to perform the same verification on a Java implementation of the transformation, we would require equally rich class and operation contracts for, say, Ecore in JML [21]. To the best of our knowledge, no research has explored automatically inferring such contracts. Even then, we expect that the user would have to explicitly specify loop invariants as soon as the transformation contains non-trivial loops, like the loops in our transformation.

Second, the study translates a substantial subset of ATL for verification, i.e., all rules except for imperative blocks, recursive lazy rules and recursive query operations other than relational closures. Thus, the approach takes advantage of the ways declarative, rule-based transformation languages (e.g., ATL) provide to iterate over the input model without requiring recursion or looping. This simplifies verification by, for instance, obviating the need for loop invariants. Although this subset of ATL is not Turing-complete, it can be used to implement many non-trivial transformations. We have statically checked the 131 transformations (comprising 2825 individual rules) in the ATL transformation zoo [36], and 83 of them fall into the described fragment, i.e., neither use recursive rules nor imperative features. Of the remaining 48 transformations, 24 of them that use imperative blocks but no recursion could be expressed declaratively, too.

We conclude that our verification approach greatly benefited from the conceptual simplicity of the declarative fragment of ATL compared to, e.g., a generalpurpose programming language such as Java.

\subsection{Limitations of the Verification Approach}

We identify two limitations of the verification approach.

Correctness of ATL-to-relational-logic translation: Extensive testing and inspection was used to ensure that all steps involved in the translation of ATL and OCL to first-order relational logic are correct. However, in the absence of a formal semantics of ATL and OCL, a formal correctness proof is impossible and the possibility of a bug in the translation remains. This should be taken into account before our approach is used in the context of safety-critical systems.

Bounded search approach: All verification approaches based on a bounded search space cannot guarantee correctness of a transformation because the scopes experimented with may have been too small. The maximum scope sufficient to show bugs in a transformation is transformation-dependent. For example, a transformation with a multiplicity invariant that requires a multiplicity to be 10 , will require a scope of 11 to generate a counterexample for that invariant, if any. With respect to our case study, we are confident that a scope of 5 is sufficient to detect violations of the given constraints; we ran analyses with scopes up to 12, because we wanted to study the performance of the approach. Real proofs of unsatisfiability can be created using SMT solvers and quantifier reasoning [9], but the problem is generally undecidable (i.e., the SAT solver does 
not terminate on all transformations), and the mapping presented in [9] does not yet cover all language features used in our case study. Further, we have not yet applied any a priori optimizations of the search problem, e.g., metamodel pruning [30], which we plan to apply for future work.

\section{Related Work}

There are several approaches that translate declarative model transformations into some logic or logic-like language to perform automated verification. Anastasakis et al. [3] and Baresi and Spoletini [5] use relational logic and the Alloy analyzer to check for inconsistencies in a transformation. Inaba et al. [19] verify the typing of transformations with respect to a metamodel using second-order monadic logic and the MONA solver. Troya and Vallecillo [34] define an encoding of ATL in rewriting logic, that can be used to check the possible executions of a transformation in Maude. Cabot et al. [11] translated QVT-R and triple graph grammar transformations into OCL contracts, requiring an OCL model finder to conduct the counterexample checking. Our translation of ATL into OCL (based on [10]) closely resembles this approach. In another previous work [9], we have presented a mapping of ATL directly into first-order logic, using quantifier reasoning to prove transformation properties with SMT solvers.

Asztalos et al. [4] formulated transformations and their properties as assertions in first-order logic. A deduction system was implemented to deduce the properties from the rules. Lucio et al. [24] verified correctness constraints for transformations in DSLTrans language using a model checker implemented in Prolog. Rensink [28] checked first-order linear temporal properties for graph transformation systems. Becker et al. [6] verified a metamodel refactoring implemented as a graph rewriting system by extending the metamodel with predicate structures which were used to specify well-formedness graph constraints. Stenzel et al. [31] implemented an algebraic formalization of a subset of operational QVT in the KIV theorem prover.

There are also several approaches that use OCL constraints to specify contracts for model transformations. Guerra et al. [18], Gogolla and Vallecillo [16], Braga et al. [7], and Cariou et al. [12] discussed testing transformations against contracts. In the same vein, Narayanan et al. [26] discuss a methodology to specify structural correspondence rules between source and target. Our constraints presented in Sect. 4.2 can be considered a transformation contract in this sense, although we do not use the contracts to test the actual transformation implementation but use them to verify the transformation independent of any input.

Regarding the used approach to check the satisfiability of OCL-constrained models, there are several potential alternatives to the USE Model Validator [23] that we employed. Gonzalez et al. [17] implemented the EMFtoCSP model finder that encodes metamodels and OCL constraints as constraint-logic programs (performing bounded verification). Queralt and Teniente [27] implemented a symbolic reasoning procedure for OCL constraints, based on predicate calculus. Brucker et al. [8] implemented the HOL-OCL theorem prover to interactively 
prove correctness constraints. Jackson et al. [20] used the FORMULA tool to reason about metamodels, but they did not support OCL.

The novel aspect of our study is two-fold: First, we have applied an automated verification methodology to an industrial model transformation implemented in the ATL transformation language. Second, we have shown the applicability of this approach to realistic search spaces and discussed the performance of our approach. Most of the referenced research papers evaluate their verification approach on small examples and do not address the performance aspect.

\section{Conclusion and Future Work}

In this study, we demonstrated how automated verification can be useful in verifying industrial transformations. First, we described the GM-to-AUTOSAR transformation that we have developed for General Motors [29]. We also discussed an automated transformation verification prototype that works on the declarative, non-recursive subset of ATL and its application to our transformation. The prototype was able to uncover two bugs in the transformation that violated two multiplicities in the AUTOSAR metamodel. We further discussed the performance of the verification prototype by showing the translation and constraint solving times for all the constraints over different scopes. The numbers showed that both the Translation times and the Constraint Solving times grow exponentially with the scope. Nonetheless, analysis of the transformation in sufficiently large scopes (up to 12) was possible. We conclude that the application of our verification approach to the case study was successful and provides evidence for its practicality, even in industrial contexts.

For future work, this study can be extended in several ways. First, other industrial transformations should be incorporated in the case study to have a better idea of the practicality of using automated verification on such transformations. Our case study explored a transformation that manipulates metamodels that are considered large on an industrial scale. The transformation, although far from being trivial, does not fully manipulate the two metamodels. We conducted a couple of experiments that show that the verification problem scales almost linearly when more independent rules are added. However, we still need to investigate the performance on larger and more complex transformations. As a result of our demonstration of the effectiveness of our approach in migrating a subset of the GM metamodel to its AUTOSAR equivalent, engineers at General Motors have expressed interest in extending the transformation to the full scope of the GM metamodel. Second, incremental SAT solvers can be used in the bounded search approach to improve the performance and the execution time of the approach, as suggested in Section 5.2. Third, pruning of the manipulated metamodels or the transformation model can be applied before executing the bounded search, as suggested in Section 6.2. 


\section{References}

1. AUTOSAR Consortium. AUTOSAR System Template, http://AUTOSAR.org/index.php? $p=3 \& u p=1 \& u u p=3 \& \quad$ uuup $=3 \& u u u u p=0 \&$ uuuuup=0/AUTOSAR_TPS_SystemTemplate.pdf, 2007.

2. AUTOSAR Consortium. AUTOSAR, http://AUTOSAR.org/, 2007.

3. K. Anastasakis, B. Bordbar, and J. Küster. Analysis of Model Transformations via Alloy. MoDeVVa, pages 47-56, 2007.

4. M. Asztalos, L. Lengyel, and T. Levendovszky. Towards Automated, Formal Verification of Model Transformations. In ICST, pages 15-24, Paris, France, 2010.

5. L. Baresi and P. Spoletini. On the Use of Alloy to Analyze Graph Transformation Systems. In ICGT, volume 4178 of $L N C S$, pages 306-320, 2006.

6. B. Becker, L. Lambers, J. Dyck, S. Birth, and H. Giese. Iterative Development of Consistency-Preserving Rule-Based Refactorings. ICMT, pages 123-137, 2011.

7. C. Braga, R. Menezes, T. Comicio, C. Santos, and E. Landim. On the Specification, Verification and Implementation of Model Transformations with Transformation Contracts. In $S B M F$, volume 7021 of $L N C S$, pages 108-123, 2011.

8. A. D. Brucker and B. Wolff. Semantics, Calculi, and Analysis for Object-Oriented Specifications. Acta Informatica, 46(4):255-284, 2009.

9. F. Büttner, M. Egea, and J. Cabot. On Verifying ATL Transformations Using Off-the-Shelf SMT Solvers. In MODELS, volume 7590 of $L N C S$, pages 432-448, 2012.

10. F. Büttner, M. Egea, J. Cabot, and M. Gogolla. Verification of ATL Transformations Using Transformation Models and Model Finders. In ICFEM, volume 7635 of LNCS, pages 198-213, 2012.

11. J. Cabot, R. Clarisó, E. Guerra, and J. de Lara. Verification and Validation of Declarative Model-to-Model Transformations Through Invariants. Systems and Software, 83(2):283-302, 2010.

12. E. Cariou, N. Belloir, F. Barbier, and N. Djemam. OCL Contracts for the Verification of Model Transformations. EASST, 24, 2009.

13. T. Cottenier, A. Van Den Berg, and T. Elrad. The Motorola WEAVR: Model Weaving in a Large Industrial Context. In $A O S D$, volume 32, Vancouver, Canada, 2007.

14. A. Daghsen, K. Chaaban, S. Saudrais, and P. Leserf. Applying Holistic Distributed Scheduling to AUTOSAR Methodology. In ERTSS, Toulouse, France, 2010.

15. H. Giese, S. Hildebrandt, and S. Neumann. Model Synchronization at Work: Keeping SysML and AUTOSAR Models Consistent. Graph Transformations and Model-Driven Engineering, 5765:555-579, 2010.

16. M. Gogolla and A. Vallecillo. Tractable Model Transformation Testing. In ECMFA, pages 221-236, Birmingham, UK, 2011.

17. C. A. González Pérez, F. Büttner, R. Clarisó, and J. Cabot. EMFtoCSP: A Tool for the Lightweight Verification of EMF Models. In FormSERA, pages 44-50, Zurich, Switzerland, 2012.

18. E. Guerra, J. de Lara, M. Wimmer, G. Kappel, A. Kusel, W. Retschitzegger, J. Schönböck, and W. Schwinger. Automated Verification of Model Transformations Based on Visual Contracts. Automated Software Engineering, 20(1):5-46, 2013.

19. K. Inaba, S. Hidaka, Z. Hu, H. Kato, and K. Nakano. Graph-Transformation Verification Using Monadic Second-Order Logic. In PPDP, pages 17-28, 2011. 
20. E. Jackson, T. Levendovszky, and D. Balasubramanian. Automatically reasoning about metamodeling. SoSyM, pages 1-15, 2013.

21. B. Jacobs and E. Poll. A Logic for the Java Modeling Language JML. In FASE, volume 2029 of $L N C S$, pages 284-299, 2001.

22. F. Jouault, F. Allilaire, J. Bézivin, and I. Kurtev. ATL: A Model Transformation Tool. Sci. Comput. Program., 72(1-2):31-39, 2008.

23. M. Kuhlmann, L. Hamann, and M. Gogolla. Extensive Validation of OCL Models by Integrating SAT Solving into USE. In TOOLS, volume 6705 of $L N C S$, pages 290-306, 2011.

24. L. Lúcio, B. Barroca, and V. Amaral. A Technique for Automatic Validation of Model Transformations. MODELS, pages 136-150, 2010.

25. P. Mohagheghi and V. Dehlen. Where is the Proof?-A Review of Experiences from Applying MDE in Industry. In ECMDA-FA, pages 432-443, 2008.

26. A. Narayanan and G. Karsai. Verifying Model Transformations by Structural Correspondence. EASST, 10(0), 2008.

27. A. Queralt and E. Teniente. Verification and Validation of UML Conceptual Schemas with OCL Constraints. TOSEM, 21(2):13, 2012.

28. A. Rensink. Explicit State Model Checking for Graph Grammars. In Concurrency, Graphs and Models, volume 5065 of LNCS, pages 114-132, 2008.

29. G. Selim, S. Wang, J. Cordy, and J. Dingel. Model Transformations for Migrating Legacy Models: An Industrial Case Study. ECMFA, pages 90-101, 2012.

30. S. Sen, N. Moha, B. Baudry, and J.-M. Jézéquel. Meta-model Pruning. In MODELS, volume 5795 of $L N C S$, pages 32-46, 2009.

31. K. Stenzel, N. Moebius, and W. Reif. Formal Verification of QVT Transformations for Code Generation. MODELS, pages 533-547, 2011.

32. M. Tisi, F. Jouault, P. Fraternali, S. Ceri, and J. Bézivin. On the Use of HigherOrder Model Transformations. In ECMDA-FA, volume 5562 of $L N C S, 2009$.

33. E. Torlak and D. Jackson. Kodkod: A Relational Model Finder. In TACAS, volume 4424 of $L N C S, 2007$.

34. J. Troya and A. Vallecillo. A Rewriting Logic Semantics for ATL. Journal of Object Technology, 10:5: 1-29, 2011.

35. The USE Validator. available online, http://sourceforge.net/projects/useocl/files/ Plugins/ModelValidator/.

36. The ATL Transformation Zoo. available online, http://www.eclipse.org/atl/atlTransformations/. 